\title{
Influence of Cutting Speed in Turning and Force in Subsequent Diamond Smoothing on Magnetic Properties of Steel 100Cr6
}

\author{
Oliver Maurer ${ }^{1, * \mathbb{D}}$, Hendrik Liborius ${ }^{2, *}$, Christiane Rauch ${ }^{1}$, Dirk Bähre ${ }^{1,3}$ and Andreas Schubert ${ }^{2} \mathbb{( 1 )}$ \\ 1 Institute of Production Engineering, Saarland University, 66123 Saarbrücken, Germany; \\ christiane.rauch@uni-saarland.de (C.R.); d.baehre@mx.uni-saarland.de (D.B.) \\ 2 Professorship Micromanufacturing Technology, Chemnitz University of Technology, \\ 09126 Chemnitz, Germany; andreas.schubert@mb.tu-chemnitz.de \\ 3 Center for Mechatronics and Automation Technologies (ZeMA) gGmbH, 66121 Saarbrücken, Germany \\ * Correspondence: oliver.maurer@uni-saarland.de (O.M.); hendrik.liborius@mb.tu-chemnitz.de (H.L.); \\ Tel.: +49-681-302-2014 (O.M.)
}

check for updates

Citation: Maurer, O.; Liborius, H.; Rauch, C.; Bähre, D.; Schubert, A Influence of Cutting Speed in Turning and Force in Subsequent Diamond Smoothing on Magnetic Properties of Steel 100Cr6. Metals 2021, 11, 1131. https://doi.org/10.3390/met11071131

Academic Editor: Jiro Kitagawa

Received: 14 June 2021

Accepted: 14 July 2021

Published: 16 July 2021

Publisher's Note: MDPI stays neutral with regard to jurisdictional claims in published maps and institutional affiliations.

Copyright: (c) 2021 by the authors. Licensee MDPI, Basel, Switzerland. This article is an open access article distributed under the terms and conditions of the Creative Commons Attribution (CC BY) license (https:// creativecommons.org/licenses/by/ $4.0 /)$.

\begin{abstract}
Magnetic properties are known to be crucial in the application of electrical steel and they are therefore covered by manifold studies. Other ferromagnetic materials are out of scope in this respect, even if the importance of magnetism of conventional steel is evident. Additionally, there is a contradiction regarding the major influence on magnetic properties. Machining, transport, and storage are possible influencing variables. In the experimental investigations, specimens consisting of the bearing steel $100 \mathrm{Cr} 6$ are machined by turning and partly by subsequent diamond smoothing. While machining using several cutting speeds and smoothing forces, the thermoelectrical voltage, current, and the components of the resultant force are recorded. The results show how the nearsurface plastic deformations evolve throughout the machining process. Additionally, it was found that the magnetic properties and other properties of the surface layer are influenced in different ways depending on turning and diamond smoothing parameters. Correlations between in situ and ex situ measured values are shown. This study aims to solve the aforementioned question by quantification of machining impacts of cutting speed in turning and force in diamond smoothing and its dependence on transport and storage.
\end{abstract}

Keywords: diamond smoothing; magnetic properties; scanning hall probe microscopy; turning; 3MA

\section{Introduction}

Magnetic properties of ferromagnetic materials change during the manufacturing processes of parts. The influences vary depending on the class of process, such as forming or machining, and they are further influenced by the process parameters and subsequently by deformation degree or depth of cut, for instance [1-3]. This is important for part cleanliness due to adhering chips and other particles in the manufacturing process [2,3].

From another point of view, there is a paradigm shift within the research field of manufacturing technology and production science. This evolution is elaborately described by Tekkaya et al. in metal forming [4]. The aim is to implement comprehensive characterizations into the manufacturing process, which enables to predict and control part properties by adjusting production processes and manufacturing chains. This idea is continued by Brinksmeier et al. [5] on a more microscopic scale in the concept of process signatures. A material changes its shape and properties as a result of external constraints, but the origin of these constraints is insignificant. That means, it is irrelevant whether a material is formed or machined or if it is finished by turning or milling. The material's reaction is a result of more conceptional characteristics, such as forces and stresses or plastic deformations of the surface layer, which still allows different magnitudes in the influences depending on its origin. Joining the paradigm shift, process signatures, and magnetic manipulation together 
leads to the gap of knowledge being addressed by this study. One goal is to contribute to a holistic understanding of manufacturing processes and their effects on produced parts.

Magnetic properties of steel after machining are commonly investigated for purposes of non-destructive testing (NDT) or metal forming processes. Only a few studies were carried out focusing on magnetic variations induced by machining [2,3]. Cutting technologies are almost exclusively considered in the field of Fe-Si alloys, also known as electrical steels [6-9]. From a solid-state physics point of view, mechanical stress and plasticity within a specimen affect its magnetic state the most by numeric variation of several magnetic characteristics and by deforming hysteresis loops $[10,11]$. This is used in NDT to assess residual or externally applied stresses [12,13], plastic zones, or mechanical characterizations of ferromagnetic specimens. NDT also explains the significance of different magnetic amounts or field components, which sense changes in other part properties such as microstructure or plastic deformation. Scanning hall probe microscopy (SHPM) for instance provides a three-dimensional vector of magnetic leakage flux $B$ in each point of measurement.

Since there is a shear zone in conventional machining technologies on a microscopic level, plastic deformation is possible within the surface layer [14]. This links the impact of machining on magnetic properties to the impact of metal forming on magnetic properties. Diamond smoothing (DS) also induces plastic deformations of the surface layer. The findings of the studies addressing magnetic properties of steel sheets after rolling emphasize the importance of the microstructure. Depending on the degree of deformation, variations in texture, grain size, and grain orientation are observed when magnetic properties are also affected $[1,15,16]$. Consequently, the aforementioned gap in research shrinks, if all related fields of research are considered.

\section{Materials and Methods}

In the experimental investigation, stepped cylindrical specimens consisting of the bearing steel $100 \mathrm{Cr} 6$ were used. The material was heat-treated on spheroid cementite. The geometry of the specimens is shown in Figure 1. The stage with the smallest diameter $(25 \mathrm{~mm})$ and a length of $20 \mathrm{~mm}$ was used for clamping the specimens while machining. The second stage, characterized by a diameter of $35 \mathrm{~mm}$ and a length of $10 \mathrm{~mm}$, was used as a contact area for the measurement of the thermoelectrical (Seebeck) current and voltage. The biggest stage of the specimens (length: $40 \mathrm{~mm}$, diameter: $50 \mathrm{~mm}$ ) was machined in the experimental investigations by turning and diamond smoothing.

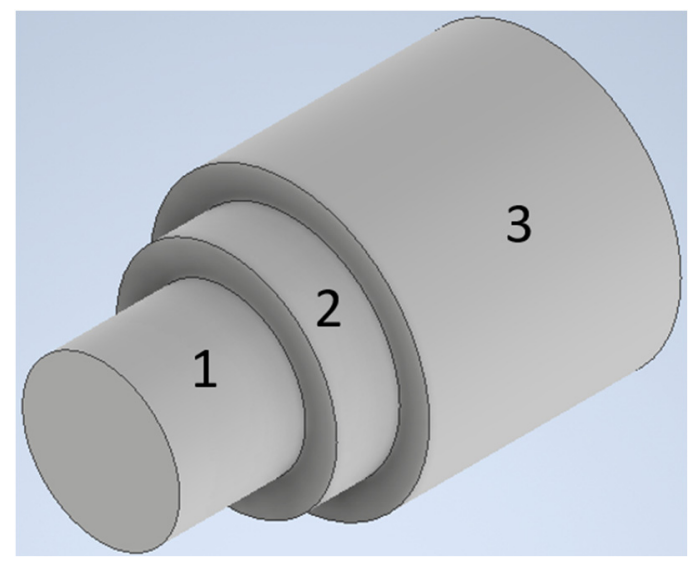

Figure 1. Specimen geometry with several stages for gripping (1), in-process temperature measurement (2), and machining experiments (3).

For the finish machining by turning, CBN (cubic boron nitride)-tipped indexable inserts of the type CCGW 09T304, characterized by a rake angle of $-20^{\circ}$ and a cutting edge rounding of about 20 to $25 \mu \mathrm{m}$, were used. The cutting material consists of $55-60 \%$ boron nitride with a grain size of about $3 \mu \mathrm{m}$, bounded by TiN. In relation to the tool holder 
used, the cutting-edge angle of the minor cutting edge was $5^{\circ}$. For diamond smoothing, a spherical body consisting of MCD (monocrystalline diamond) with a radius of $2 \mathrm{~mm}$ was utilized.

The experimental investigations were carried out on a precision lathe SPINNER type PD 32. The specimens were clamped using a dead-length collet chuck. Pre-machining of the biggest part of the specimens (3) was performed by turning to a diameter of $44.4 \mathrm{~mm}$. Finish machining was also performed by external cylindrical turning and partly by subsequent diamond smoothing, according to Table 1 . In the turning experiments, the influence of three different cutting speeds $(50,250,450 \mathrm{~m} / \mathrm{min})$ was analyzed. The feed $(0.05 \mathrm{~mm})$ and the depth of cut $(0.2 \mathrm{~mm})$ were kept unchanged. The investigations were carried out without using any cooling lubricant. The manufacturer of the tools recommends a cutting speed of 70 to $170 \mathrm{~m} / \mathrm{min}$. However, these recommendations should lead to a tool life of about $15 \mathrm{~min}$ and do not completely regard the workpiece material. The objective of the experimental investigations was to change the temperature in the shear zone as well as plastic deformation due to thermal softening. Geometrical surface properties and tool life-time are only regarded secondarily.

Table 1. Experimental setup.

\begin{tabular}{|c|c|c|c|}
\hline \multirow{2}{*}{ Experiment Number } & \multicolumn{2}{|c|}{ Turning } & \multirow{2}{*}{$\begin{array}{c}\text { Diamond Smoothing } \\
\text { Force }(\mathrm{N})\end{array}$} \\
\hline & Cutting Speed (M/Min) & Tool Number & \\
\hline $1-3$ & \multirow{2}{*}{50} & 1 & \\
\hline $4-6$ & & 2 & \\
\hline $7-9$ & \multirow{2}{*}{250} & 3 & \\
\hline $10-12$ & & 4 & \\
\hline $13-15$ & \multirow{2}{*}{450} & 5 & \\
\hline $16-18$ & & 6 & \\
\hline $19-21$ & \multirow{4}{*}{250} & 7 & \multirow{2}{*}{50} \\
\hline $22-24$ & & 8 & \\
\hline $25-27$ & & 9 & \multirow{2}{*}{150} \\
\hline $28-30$ & & 10 & \\
\hline
\end{tabular}

For subsequent diamond smoothing, specimens machined by turning with a cutting speed of $250 \mathrm{~m} / \mathrm{min}$ were used. In the diamond smoothing experiments, the influences of two different smoothing forces ( 50 and $150 \mathrm{~N}$ ) were regarded. The smoothing speed $(70 \mathrm{~m} / \mathrm{min})$ and the feed $(0.05 \mathrm{~mm})$ were kept unchanged. To improve the sliding behavior and to avoid materials' adherence between the tool and the workpiece, the emulsion flood cooling strategy was used.

Each of the five experimental combinations $\left(3 \times v_{\mathcal{c}}, 2 \times F\right)$ was repeated five times. Hence, six specimens were machined with the same parameters. To reduce the influence of the tool wear on the experimental results, the indexable inserts were changed after machining at least three specimens with the same parameters.

While finish machining (turning or diamond smoothing), the components of the resultant force were detected by a dynamometer (Kistler type 9257), on which the tool holder was mounted. Additionally, thermoelectrical (Seebeck) voltage and current were measured. For this purpose, the indexable insert was connected by a clamped cable. The rotating specimen was connected by two copper-graphite brushes at part 2 (diameter $35 \mathrm{~mm}$, length $10 \mathrm{~mm}$ ). To measure the Seebeck voltage and current, an electrical insulation of the tool and the specimen was necessary. This was realized by a sleeve consisting of polyoxymethylene (POM), which was mounted at the outer diameter of part 1 (diameter $20 \mathrm{~mm}$, length $20 \mathrm{~mm}$ ) of the workpiece. Hence, the specimen and the dead-length collet chuck were not electrically connected. Additionally, an adhesive foil was mounted at the outer surface of the tool holder. So, there was no electrical connection between the tool and the dynamometer. 
After machining, the geometrical surface properties of all specimens were detected by a stylus instrument Mahr type LD 120. The stylus used was characterized by a radius of $2 \mu \mathrm{m}$ and an angle between the stylus and the specimen rotation axis of $90^{\circ}$. The measurements were realized in the direction of feed motion with a measuring length of $4 \mathrm{~mm}$. Filtering of the profiles was carried out in accordance with DIN 11562. Five single measurements evenly distributed at the perimeter were performed for each specimen. Additionally, the machined surfaces of all specimens were analyzed by 3D laser scanning microcopy using a Keyence type VK-9700. The measured quadratic field had a lateral length of $0.5 \mathrm{~mm}$. For each machined specimen, two such measurements were performed.

Every specimen was inspected with scanning hall probe microscopy (SHPM, Metrolab 1176LF probe) in a continuous dynamic mode. Measurements were performed along a scanning axis in positive x-direction in 8 different orientations of $\varphi$ (steps of $45^{\circ}$ each, see Figure 2a). Some specimens were prepared for microstructural analysis by grinding and polishing. The spot specimens are taken from is sketched in Figure 2b. These specimens were checked in digital microscopy (Keyence VHX-7000) for their purity grade, in magnetic force microscopy (MFM, Bruker) for their structure of magnetic domains, and after etching in alcoholic nitric acid again in digital microscopy for microstructural assessment. Residual stress states had been evaluated in XRD experiments (PANalytical Empyrean) using the $\sin ^{2} \varphi$-method. This method puts out normal and shear components of residual stresses in tangential direction and feed direction. No aforementioned technique changed the magnetic state of a specimen. Possible effects had to result from machining, transport, or storage.

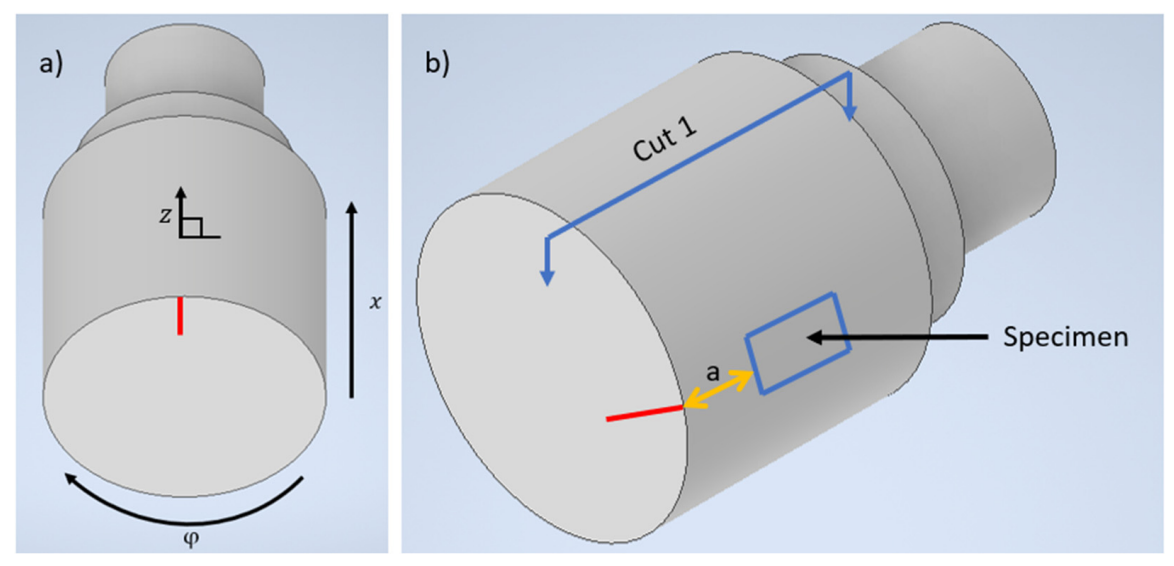

Figure 2. Specimens (a) definition of the coordinates and (b) location of the metallographic specimen. $0^{\circ}$ of SHPM measurements marked in red.

Additionally, micromagnetic measurements were performed using a 3MA sensor with a probe that was adjusted to the specimen diameter. One specimen of each parameter set was inspected by 3MA at several spots of measurement. 3MA uses Magnetic Barkhausen Noise (MBN), harmonic analysis, incremental permeability, and eddy current at the same time, and measures up to 71 characteristics at once, which mostly characterize a sample's surface. Due to micromagnetic tests, the magnetic properties of a specimen were changed irreversibly, so there is no opportunity for further investigations of manufacturing effects.

Several data processing techniques, such as image processing (Gwyddion for MFM micrographs), correlation, and score calculation, were applied to the signals measured. A Matlab-based mapping tool that sketches magnetic leakage fluxes in pictures for every flux vector component and the vector magnitude of SHPMed specimens was created.

The impact of transport and storage on magnetic part properties had to be assessed using inspection methods, by which the magnetic state of the specimen remains in pristine conditions. This is why SHPM was used. Two different types of parcels were shipped from one institute to the other. In type 1 shipping, specimens were shielded from each other 
and the whole parcel was shielded from its environment, so specimens could not influence each other by friction, for instance, nor were they affected by electromagnetic radiation on their journey. In type 2 shipping, these actions had not been taken. Specimens were covered in bubble wrap to avoid damages only. Different types, several steps of shipping, and consecutive SHPM measurements of every specimen allow to determine transport effects, as follows:

$$
\begin{gathered}
\left.\Delta B_{\mathrm{T}}(\mathrm{n}=1)=\bar{B} \text { (Type1 shipping }\right)-\bar{B} \text { (Type2 shipping) } \\
\left.\left.\Delta B_{\mathrm{T}}(\mathrm{n}=2)=\bar{B} \text { (multiple shipping }\right)-\bar{B} \text { (Type2 shipping }\right) \\
\left.\Delta B_{\mathrm{T}}(\mathrm{n}=3)=\bar{B} \text { (multiple shipping }\right)-\bar{B} \text { (Type1 shipping) }
\end{gathered}
$$

where $\Delta B_{\mathrm{T}}$ describes the impacts of transport depending on the amount of shipping cycles $\mathrm{n}$ and $\bar{B}$ is the mean value of leakage flux of a certain shipping mode. Multiple shipped samples were sent back and forth between the institutes three times. A comparison of effects caused by machining and transportation requires mathematical definitions of machining effects, $\Delta B_{\mathrm{M}}$, and storage effects, $\Delta B_{\mathrm{S}}$, because specimens were stored for several days in addition to transportation:

$$
\begin{gathered}
\Delta B_{\mathrm{M}}=\bar{B}(\text { Type1 shipping })-\bar{B}(\text { premachining }) \\
\Delta B_{\mathrm{S}}(\Delta \mathrm{t})=\bar{B}(\text { stored })-\bar{B}(\text { initial })
\end{gathered}
$$

Results of the comparison of machining and transportation were chosen to be expressed by a quotient $Q(n) \in R$, which is determined by the following equation:

$$
Q(n)=\left\{\begin{aligned}
\frac{\Delta B_{T}}{\Delta B_{M}}, & n=1 \\
\frac{\Delta B_{T}-\Delta B_{S}}{\Delta B_{M}}, & n \in\{2 ; 3\}
\end{aligned}\right.
$$

Machining predominates transportation if $|Q(n)|<1$ stands, otherwise, if $(|Q(n)|>1)$, transportation effects are stronger than machining influences.

\section{Results}

\subsection{In-Process Measurements}

In turning investigations, the components of the resultant force are analyzed in the range of theoretical constant cross-section of undeformed chip (entry and exit of the tool and the workpiece are not regarded). In this range, the mean value of each component is calculated. The influence of the cutting speed on cutting force, feed force, and the passive force is shown in Figure 3. The cutting force acts in the cutting direction, the feed force in the direction of feed motion, and the passive force perpendicular to the workpiece surface. All three force components are oriented perpendicularly to each other.

The values shown represent the arithmetic mean values of the six single experiments performed with the same parameters. The respective minimum and maximum correspond to the highest and the lowest values of these six measurements. Regarding the turning experiments, in spite of the cutting speed, the cutting force represents the highest component, followed by the passive force and the feed force. There is a strong decrease in force components when machining with a cutting speed of 50 and $250 \mathrm{~m} / \mathrm{min}$ is compared. For 250 and $450 \mathrm{~m} / \mathrm{min}$, the decrease in force components is less distinct. Generally, a decrease of the force components is expected with increasing cutting speed due to the higher shear zone temperatures, thermal softening, and decreasing force demand for material separation. The stronger decrease of the components of the resultant force between the cutting speeds of 50 and $250 \mathrm{~m} / \mathrm{min}$ is explained by the comparatively bad working range of the cutting material at the low cutting speed $(50 \mathrm{~m} / \mathrm{min})$. Machining using CBN requires a defined temperature. Regarding the combination of workpiece, tool geometry, and cutting material, a higher temperature in the shear zone is required. The unsuitable cutting speed also leads to clamping of chips between tool and workpiece from time to time. The small 
decrease in components of resultant forces comparing the machining with cutting speeds of 250 and $450 \mathrm{~m} / \mathrm{min}$ confirms the expected difference in force components due to the thermal softening.

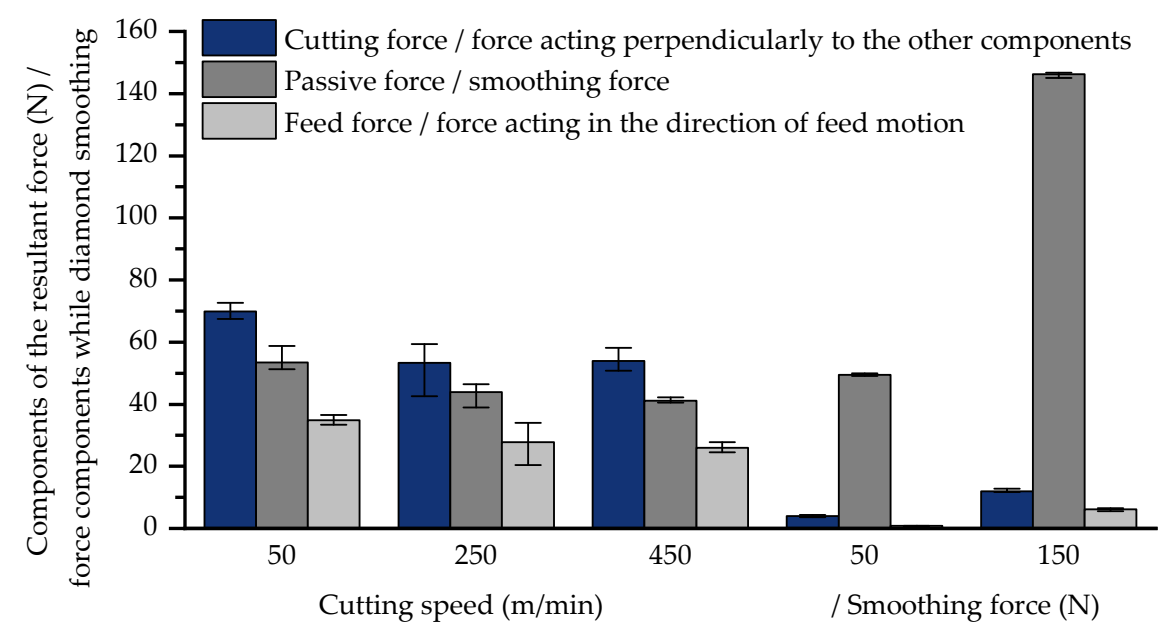

Figure 3. Influence of the cutting speed in turning on components of the resultant force and the smoothing force, and diamond smoothing on the force components.

Force components are also determined while diamond smoothing. The biggest component is represented in the direction of the specimen surface (smoothing force). For the experiments 19-24, the mean value measured was $49.5 \mathrm{~N}$. Hence, the deviation to the aspired value $(50 \mathrm{~N})$ was very small. For the experiments $25-30$, the measured mean value was $146.2 \mathrm{~N}$. The percentage deviation to the aspired value $(150 \mathrm{~N})$ is in the same range as before. Both of the other force components detected had much smaller absolute values, but also increased with the smoothing force. The measured mean values in the direction of feed motion are about 0.8 and $6.1 \mathrm{~N}$ and the forces in the perpendicular direction about 4 and $12 \mathrm{~N}$.

As a mathematical product of cutting force and cutting speed, it is possible to calculate the cutting power. While machining, this power is almost entirely transformed into heat. However, the distribution in the specimen, the chip, and the tool is simultaneously influenced. For the evaluation of temperatures in the shear zone, Seebeck voltage and Seebeck current depending on the cutting speed are visualized in Figure 4. Similar to the components of the resultant force, the values shown represent the arithmetic mean values of the six single experiments performed with the same parameters. The respective minimum and maximum correspond to the highest and the lowest values of these six measurements.

Seebeck voltage corresponds to the temperature between tool and specimen in the shear zone. Due to different Seebeck coefficients of tool and workpiece, there is an increase of temperature in the contact area (shear zone and area, where chip, which is still connected to the workpiece, slides at the rake face of the tool), which leads to an increase in Seebeck voltage. A linear rise of Seebeck voltage involves a nearly linear increase of the shear zone temperature. The method uses the same effect as in commercial thermocouples. Hence, the same temperature at the connection points between tool and measuring system as well as workpiece and measuring systems is required, to increase the accuracy of the method. To analyze the real temperatures, determination of Seebeck coefficients of the tool and the specimen material would be necessary. However, currently, this is associated with a comparatively high effort. Consequently, temperatures are not added. In the experimental investigation, regarding the tendencies should be adequate. Seebeck current is influenced by the temperature and the contact area between the tool and the specimen. Hence, it is also possible to analyze the changes in this area due to flank wear land width. Regarding the diagram, increasing the cutting speed results in a rise in Seebeck voltage and current. This corresponds to the already explained higher cutting power and shear zone temperature. 
However, the increase in Seebeck voltage is much stronger between 50 and $250 \mathrm{~m} / \mathrm{min}$ than between 250 and $450 \mathrm{~m} / \mathrm{min}$. This may be explained by larger amounts of heat in the chip with increasing cutting speed.

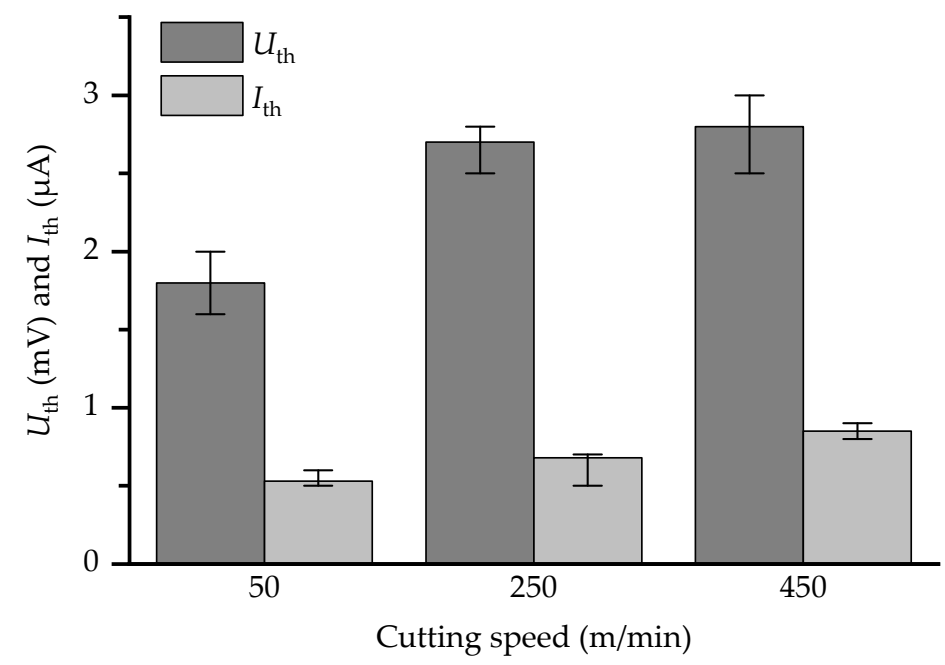

Figure 4. Influence of the cutting speed in turning on the Seebeck voltage and current.

\subsection{Geometrical Surface Properties}

The influence of the cutting speed in the turning process and the smoothing force in subsequent diamond smoothing on the arithmetic mean surface roughness, $R a$, and the surface roughness depth, $R z$, is shown in Figure 5. The mean values show the arithmetic mean values of the 30 measurements for specimens machined with the same parameters (6 specimens with respectively 5 measurements). The variations shown represent the standard deviation.

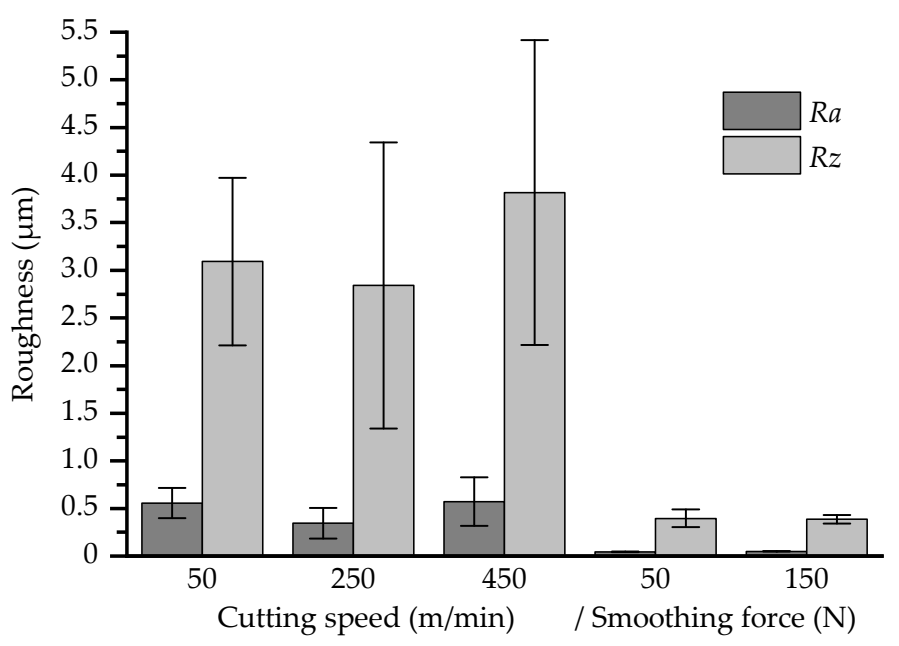

Figure 5. Influence of the cutting speed in turning and the smoothing force while diamond smoothing on $R a$ and $R z$.

Generally, the mean values after turning are higher than the theoretical kinematic roughness $(0.78 \mu \mathrm{m})$. This is a result of the partly unsuitable tool geometry (negative rake angle) as well as tool material (CBN) used for machining this alloy after the AC heat treatment. The low hardness of the workpieces in combination with the cutting material, the negative rake angle, and the high cutting-edge rounding result in the comparatively high roughness values. The tool-workpiece combination should enable to compare these results with further experiments regarding the machining of hardened $100 \mathrm{Cr} 6$. The ma- 
chining conditions while turning lead to a bad chip separation, material adherence, and subsequently increased roughness values.

Subsequent diamond smoothing results in a strong decrease of the surface roughness values and specular surfaces. For both smoothing forces analyzed, similar mean values for $R a$ and $R z$ were detected. While diamond smoothing, the peaks of roughness profiles are lowered by plastic deformation and the valleys of this profile are lifted. Depending on the initial surface properties, there is a smoothing force which leads to a minimum surface roughness. It is expected that this force is between 50 and $150 \mathrm{~N}$.

\subsection{Magnetic Characterization and the Impact of Machining}

Due to the enormous amount of data which is acquired in each measurement, especially in SHPM and 3MA, a reasonable reduction of data is advisable. In case of SHPM, two components of the magnetic leakage flux vector, namely, $\mathrm{x}$ and $\mathrm{z}$, are chosen, where $\mathrm{x}$ has the highest contrast and $\mathrm{z}$ contributes the most to the vector magnitude. Additionally, values such as maxima, mean values, and root mean square values (RMS) are calculated in order to provide an extract to feed into plots and calculation. In case of 3MA, six characteristics were chosen, namely two each of MBN signal amplitude, incremental permeability, and coercive field, including key parameters of the magnetic hysteresis.

The magnetic structure on a microscopic scale has been investigated with magnetic force microscopy. In the MFM mode, surface topography, such as in usual AFM and magnetic structure, are recorded simultaneously. Two representative results are sketched in Figure 6.

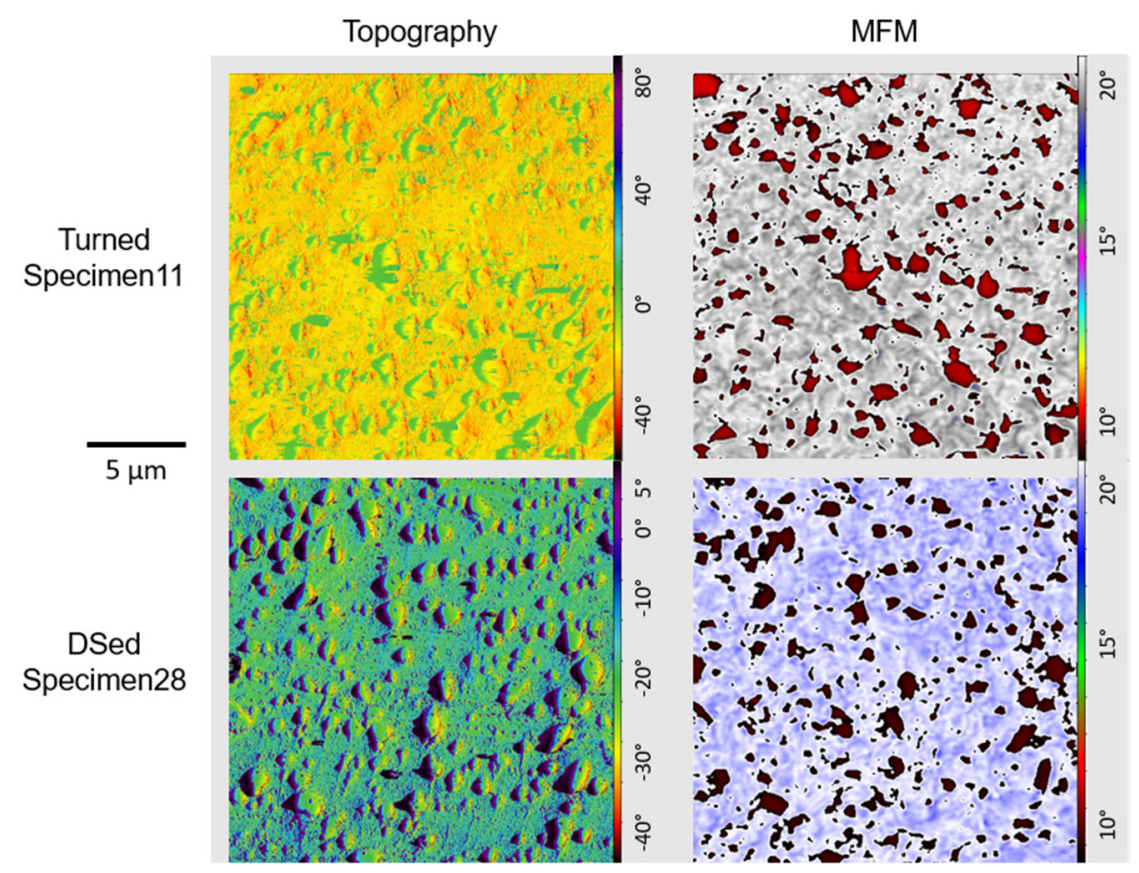

Figure 6. AFM topography and MFM images of a turned and a diamond smoothed specimen with specimen coordinates: specimen $11\left(x=9.14 \mathrm{~mm} ; \varphi=0^{\circ}\right)$ and specimen $28\left(x=15.84 \mathrm{~mm} ; \varphi=0^{\circ}\right)$.

It does not seem to matter whether a specimen is machined by turning or diamond smoothing on a scale that is as small as the MFM-inspected areas, because topography and MFM images of both specimens are very similar. It does not mean that there are no differences resulting from different machining technologies. The images represent an area of $20 \mu \mathrm{m}$ squared. Differences possibly appear on larger scales, which could not be inspected in the MFM experiments due to the instrumental limits. However, both image types are pervaded with mostly small dot-shaped features, which seem to be precipitations. MFM micrographs reveal, in a dark and quite homogeneous color scheme, that these 
precipitations must be paramagnetic. The precipitations are surrounded by a matrix in a marbled color scheme. Every micrograph taken in MFM experiments contains these marbled areas, which means that there is a ferromagnetic matrix. Additional microscopical analyses of the surface layer are shown in Figure 7.

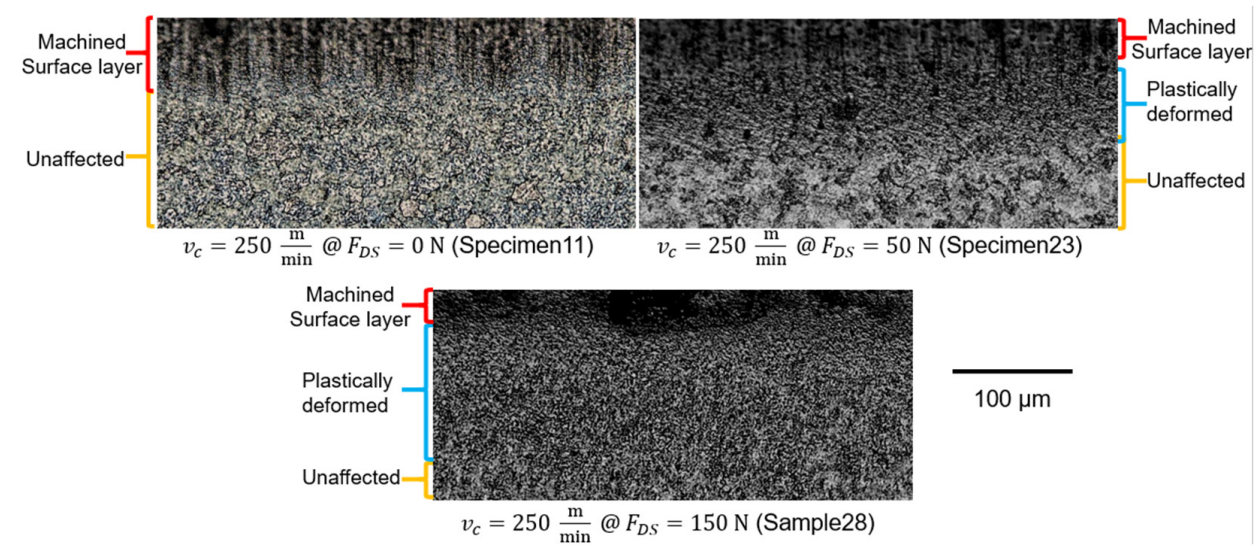

Figure 7. Microstructural identification of plastically deformed zones.

Even if MFM micrographs do not show boundaries of magnetic domains-probably due to the small inspection area-a variation of domain size, orientation, and structure still seems possible. The state-of-the-art says that plastic deformation (e.g., caused by forming) changes the magnetic domain structure. Again, metallographic analysis and digital microscopy confirm the plastic deformation on the surface and in a sub-surface shell after DS. The affected zones increase from turning to diamond smoothing and further increase in the case of diamond smoothing with higher forces.

Micromagnetic 3MA measurements reveal the impacts of turning and DS. Since 3MA combines various NDT methods, many signals are recorded at the same time. Coercive field strength is determinable via several techniques used by 3MA, so there is a special interest in comparing the coercive fields of $\mathrm{MBN}, \mathrm{H}_{\mathrm{CM}}$, and the coercive fields of incremental permeability measurements, $H_{\mathrm{C} \mu}$, with each other. Furthermore, remanent and maximal Barkhausen signals, $M_{\mathrm{R}}$ and $M_{\max }$, which are represented by MBN signal amplitudes, are evaluated. Mean and maximum relative incremental permeability, $\mu_{\mathrm{r} \text {, max }}$ and $\mu_{\mathrm{r} \text {, mean }}$, are regarded as well. Machining parameters of turning and diamond smoothing, cutting speed, and force in DS, plotted versus the experimental results of 3MA, are sketched in Figure 8. The cutting speed of $v_{\mathrm{c}}=0 \mathrm{~m} / \mathrm{min}$ means that the specimen was only pre-machined by turning.

As it can be seen in every single diagram, plots of maximum and average values of each characteristic follow almost the same mathematical law. Related characteristics and coercive fields, $H_{\mathrm{CM}}$ and $H_{\mathrm{C} \mu}$, are influenced in a very similar way. Turning causes an increase in maximum Barkhausen signal, $M_{M a x}$, and remanence signal, $M_{R}$, as the cutting speed increases. $M_{\max }$ at $v_{\mathrm{C}}=0 \mathrm{~m} / \mathrm{min}$ is larger than at $v_{\mathrm{C}}=50 \mathrm{~m} / \mathrm{min}$, so pre-machining obviously has an influence. However, a trend in the curves of magnetization is evident. That trend is supported by plots of coercive fields and relative incremental permeabilities. Independent from the method of determination, coercive fields tend to decrease as the cutting speed increases. Relative incremental permeability slightly increases over cutting speed. These effects lead to a deformed hysteresis loop, which is "thinner" and "taller" depending on the turning parameters. Therefore, an increase in cutting speed tends to soften the steel from a magnetic point of view, due to a change in the area enclosed by the hysteresis loop.

Diamond smoothing causes the countereffect of turning. Hence, magnetizations and relative incremental permeabilities decrease significantly over DS force, whereas coercive field strengths increase. The resulting hysteresis loop is also deformed, but towards a more simple and wide shape. This means that DS leads to magnetic hardening of parts. 

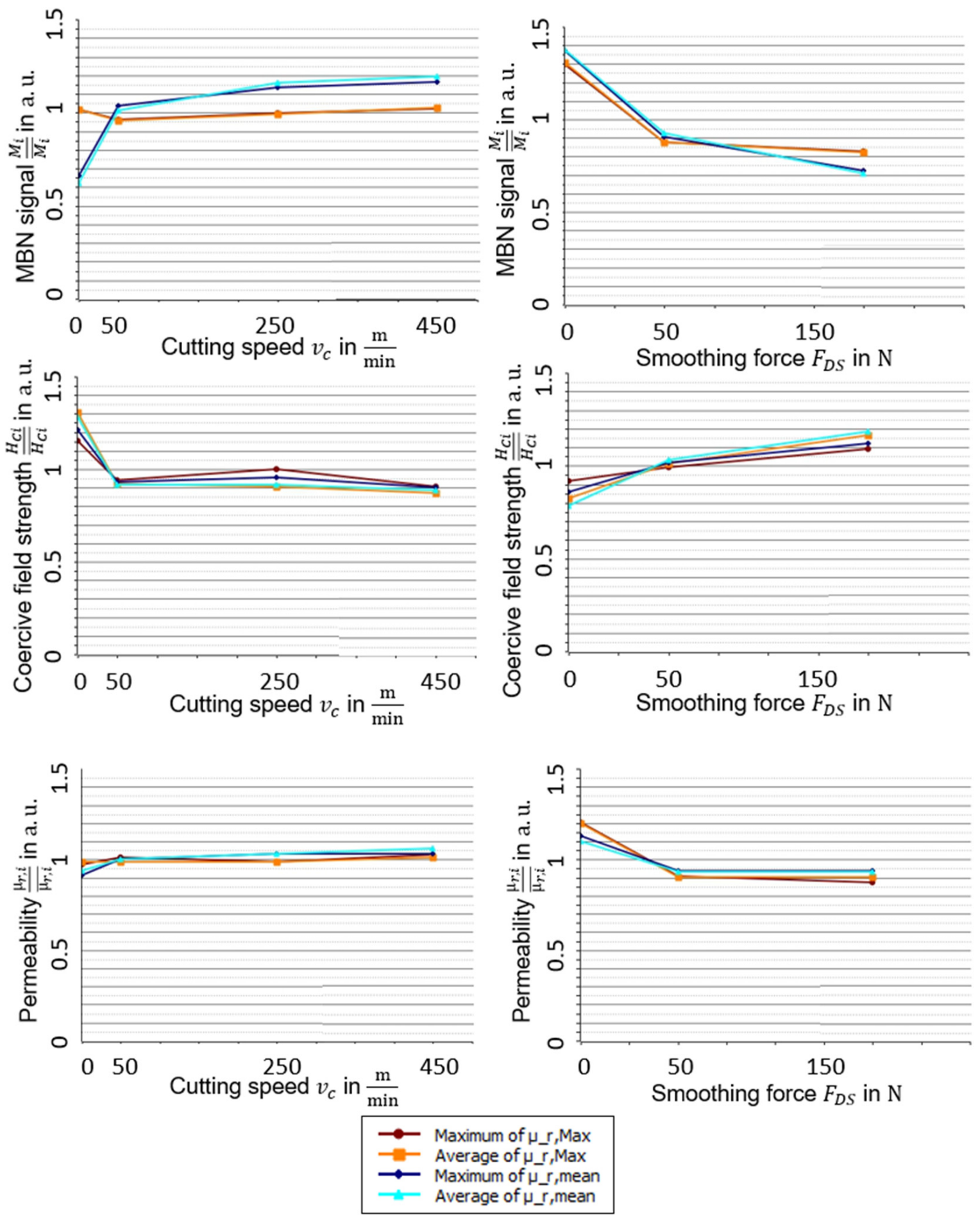

Figure 8. Dependencies of ratios of micromagnetic characteristics of MBN signal amplitude, coercive field strength, and relative incremental permeability, divided by their mean values of machining parameters. Maxima in dark color, averages in light color.

The opposed effects of turning and DS may result from different origins. On the one hand, surface layers significantly plastify by DS, when compared to turning. Discrepant deformation degrees seem to be a plausible reason, as the plastic deformations are brought into each part at room temperature (in spite of local in-process heating by friction), so cold working occurs. Since there is a correlation between magnetic and mechanical hardness, cold working and magnetic hardening as effects of DS fit together. 
SHPM measurements reveal, in addition to the other results, information about the impact of machining on magnetic leakage fluxes. Building on the foundation of SHPM, assumptions regarding the behavior of magnetic domains can also be stated, but they are not as reliable as results of MFM analysis.

The maps in Table 2 show that machining seems to increase homogeneity in magnetic leakage flux. The x-component is sketched with its absolute value because there are negative and positive values in order to establish comparability to the generally positive $\mathrm{z}$-component. After turning, there are still some hotspots, and it is unclear whether these hotspots are caused by turning or whether they are relics of the pristine condition. However, diamond smoothing burnishes those hotspots and increases the magnetic leakage fluxes in z-direction. By increasing the DS force, this effect becomes stronger.

Table 2. Magnetic leakage flux maps, $B(x, \varphi)$, including the coordinates $x(\mathrm{~mm})$ in vertical and $\varphi\left(1^{\circ}\right)$ in horizontal direction for different machining conditions.

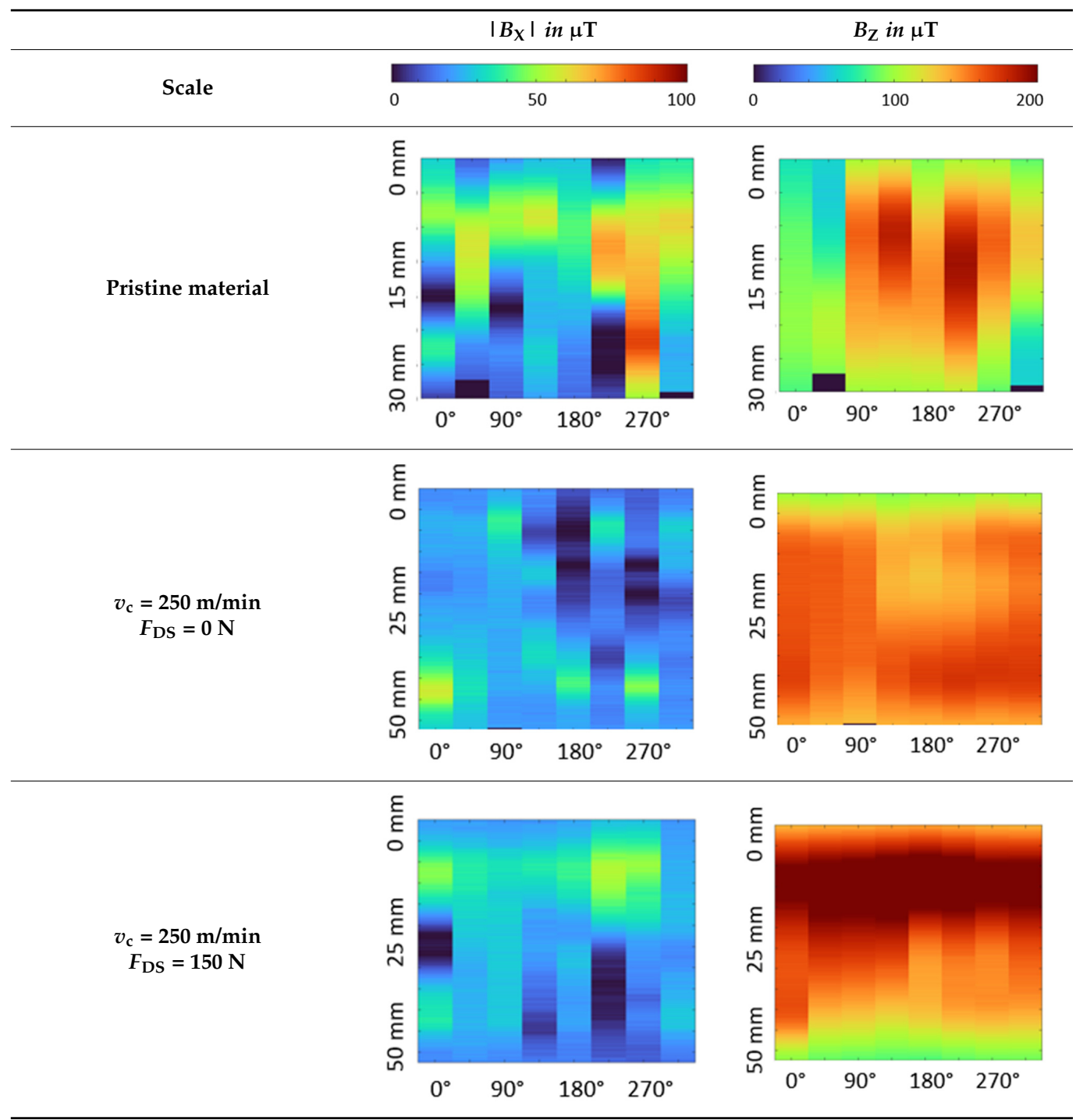

As values such as maxima, mean values, and root mean square values (RMS) were extracted from the maps, it is obvious that turning leads to higher leakage fluxes in $x$ - and $z$-directions. The effect grows stronger as the cutting speed increases. Therefore, it can be assumed that turning affects magnetic domains, which depend strongly on leakage fluxes. Hence, even if MFM imaging did not allow to assess domain structures, it can be stated that turning changes this structure. Since DS is a process of forming without producing chips, 
effects vary from those in turning. A process of magnetization occurs as well. Leakage fluxes are not as heavily manipulated compared to turning. Further experiments should clarify whether the leakage flux saturates after different stages of machining or whether the impact really is smaller than the one caused by turning.

, including the coordinates $x(\mathrm{~mm})$ in vertical and $\varphi\left(1^{\circ}\right)$ in horizontal direction for different machining conditions.

\subsection{The Impact of Transport and Storage}

SHPM is also capable of working out the impacts of transport and storage. On the one hand, differential mapping provides a visual impression of these impacts, and on the other hand, numerical differences and quotients $Q(n, B)$ (calculation is provided in Equation (6)) characterize the circumstances.

It can be seen from Table 3 that the absolute values of quotients are often $<1$, so in most cases, machining has a stronger influence than transport. Some values are $>1$, which puts emphasis on the cases where transport is stronger than machining. These characteristics are very sensitive for these influences and cannot be used to quantify the impact of machining. Maximum values do not seem to be highly controlled by shipping. Mean values are tied with machining and transport, where one half proves a supremacy of machining and the other half shows the contrary. The biggest difference between leakage fluxes $B_{x}$ and $B_{z}$ can be observed for RMS values. In $x$-direction, most quotients prove shipping to be stronger. In z-direction, machining is obviously almost in full control.

Table 3. Comparison of transport and machining effects via quotients $Q(B, n)$, transport has a stronger impact if $|Q|>1$.

\begin{tabular}{|c|c|c|c|c|c|c|c|c|}
\hline $\begin{array}{l}\text { Cutting } \\
\text { Speed } \\
\text { (M/Min) }\end{array}$ & $\begin{array}{l}\text { DS } \\
\text { Force } \\
\text { (N) }\end{array}$ & $\begin{array}{c}\text { Transport } \\
\text { Cycles }\end{array}$ & $Q\left(B_{x}^{\max }\right)$ & $Q\left(\overline{B_{x}}\right)$ & $Q\left(B_{x}^{R M S}\right)$ & $Q\left(B_{z}^{\max }\right)$ & $Q\left(\overline{B_{z}}\right)$ & $Q\left(B_{z}^{R M S}\right)$ \\
\hline 50 & 0 & $\begin{array}{l}1 \\
2 \\
3\end{array}$ & $\begin{array}{c}0.16 \\
-0.8 \\
-0.65\end{array}$ & $\begin{array}{c}-0.86 \\
-0.7 \\
-1.56\end{array}$ & $\begin{array}{c}3.29 \\
-6.06 \\
-2.77\end{array}$ & $\begin{array}{c}0.46 \\
-1.02 \\
-0.56\end{array}$ & $\begin{array}{c}-0.05 \\
0.02 \\
-0.02\end{array}$ & $\begin{array}{c}0.21 \\
-0.34 \\
-0.14\end{array}$ \\
\hline 250 & 0 & $\begin{array}{l}1 \\
2 \\
3\end{array}$ & $\begin{array}{c}0.17 \\
-0.65 \\
0.48\end{array}$ & $\begin{array}{c}-0.11 \\
-1.56 \\
1.46\end{array}$ & $\begin{array}{c}-9.6 \\
-2.77 \\
28\end{array}$ & $\begin{array}{c}0.31 \\
-0.21 \\
0.11\end{array}$ & $\begin{array}{l}-1.08 \\
-2.17 \\
-3.25\end{array}$ & $\begin{array}{c}-0.26 \\
-0.9 \\
-1.16\end{array}$ \\
\hline 450 & 0 & $\begin{array}{l}1 \\
2 \\
3\end{array}$ & $\begin{array}{c}0.49 \\
0.12 \\
0.6\end{array}$ & $\begin{array}{c}1.4 \\
0.08 \\
1.47\end{array}$ & $\begin{array}{l}-0.28 \\
-0.87 \\
-1.15\end{array}$ & $\begin{array}{c}0.14 \\
-0.46 \\
-0.32\end{array}$ & $\begin{array}{l}-0.75 \\
-0.03 \\
-0.78\end{array}$ & $\begin{array}{l}-0.36 \\
-0.28 \\
-0.64\end{array}$ \\
\hline 250 & 50 & $\begin{array}{l}1 \\
2 \\
3\end{array}$ & $\begin{array}{c}-0.56 \\
-0.22 \\
0.07\end{array}$ & $\begin{array}{l}-0.53 \\
-0.86 \\
-3.92\end{array}$ & $\begin{array}{l}-0.67 \\
-0.36 \\
-1.04\end{array}$ & $\begin{array}{c}-41.67 \\
0.56 \\
0.81\end{array}$ & $\begin{array}{c}-1.05 \\
0.32 \\
-0.27\end{array}$ & $\begin{array}{c}-0.79 \\
0.47 \\
0.18\end{array}$ \\
\hline 250 & 150 & $\begin{array}{l}1 \\
2 \\
3\end{array}$ & $\begin{array}{c}-1.11 \\
0.25 \\
0.4\end{array}$ & $\begin{array}{l}0.32 \\
1.71 \\
0.97\end{array}$ & $\begin{array}{l}-0.02 \\
-2.51 \\
-2.54\end{array}$ & $\begin{array}{c}-2.31 \\
0.01 \\
-0.25\end{array}$ & $\begin{array}{c}-0.97 \\
0.01 \\
-0.44\end{array}$ & $\begin{array}{c}-1.19 \\
0.03 \\
-0.36\end{array}$ \\
\hline
\end{tabular}

Figure 9 shows several trends of how transport changes the magnetic leakage fluxes in different directions. Most of the curves of each diagram are very similar to each other, so transport affects all the specimens in almost the same way. Machining conditions do not seem to be crucial for the impact of transportation. In x-direction, maxima and average values increase throughout the transport, whereas RMS values decrease. This suggests that negative leakage fluxes approach a demagnetized zero-flux state from below. Curves of both average and RMS values are shaped like hockey sticks. Probably, the impact of transport experiences saturation after a certain number of cycles of shipping or distance, respectively. In contrast, maxima of $B_{x}$ rise, so the magnetic state might become heterogenized by shipping. Although curves progress differently in z-direction, the behavior that is read off the diagrams is almost the same. Average and RMS values show a smoother shape than seen in the x-direction. Obviously, the z-component is demagnetized. Curves of z-maxima have an irregular shape and do not point to a specific 
effect of transportation. An improved statistical coverage might address this issue properly and might provide clarification.
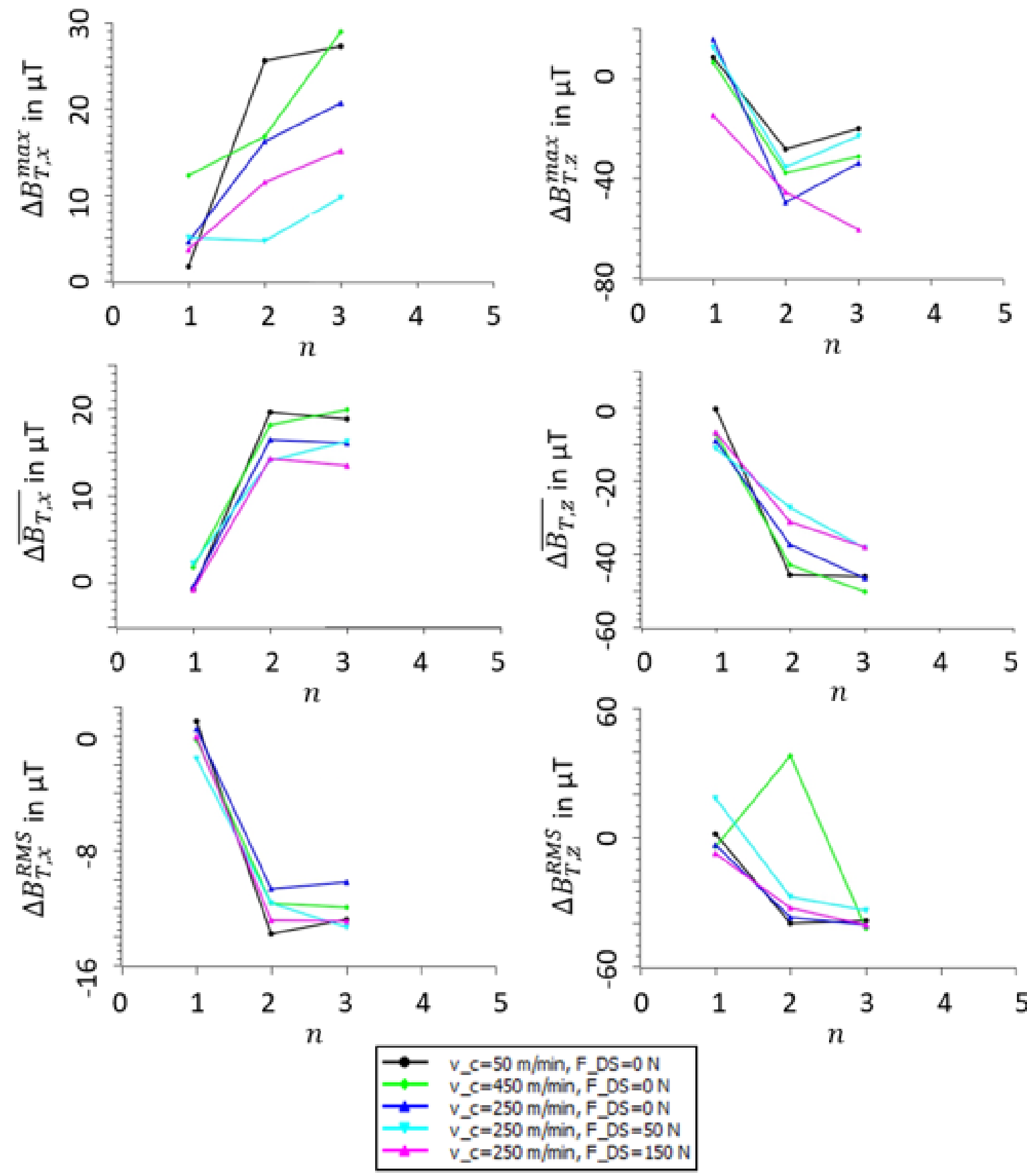

Figure 9. Changes in magnetic leakage flux components due to $n$ cycles of shipping. Calculations are performed according to Equations (1)-(3).

The influence of storage is smaller than the influence of transport. Differential imaging is used in Table 4 to illustrate how magnetic leakage fluxes evolve over a time of 44 days. Colors of the maps show that the impact of storage is nearly neglectable. Maximum change is about $15 \mu \mathrm{T}$. Leakage fluxes in $\mathrm{x}$-direction approach a demagnetized state from negative values, while z-values approach a zero-leakage flux from the positive side. Additionally, all differential maps shown are very similar to one another, so the impact of storage is considered to be independent of machining conditions and flux components. 
Table 4. Differential magnetic leakage flux maps of storage, $\Delta B_{S}(x, \varphi)$ (see Equation (5)), including the coordinates $x(\mathrm{~mm})$ in vertical and $\varphi\left({ }^{\circ}\right)$ in horizontal direction for different machining conditions.

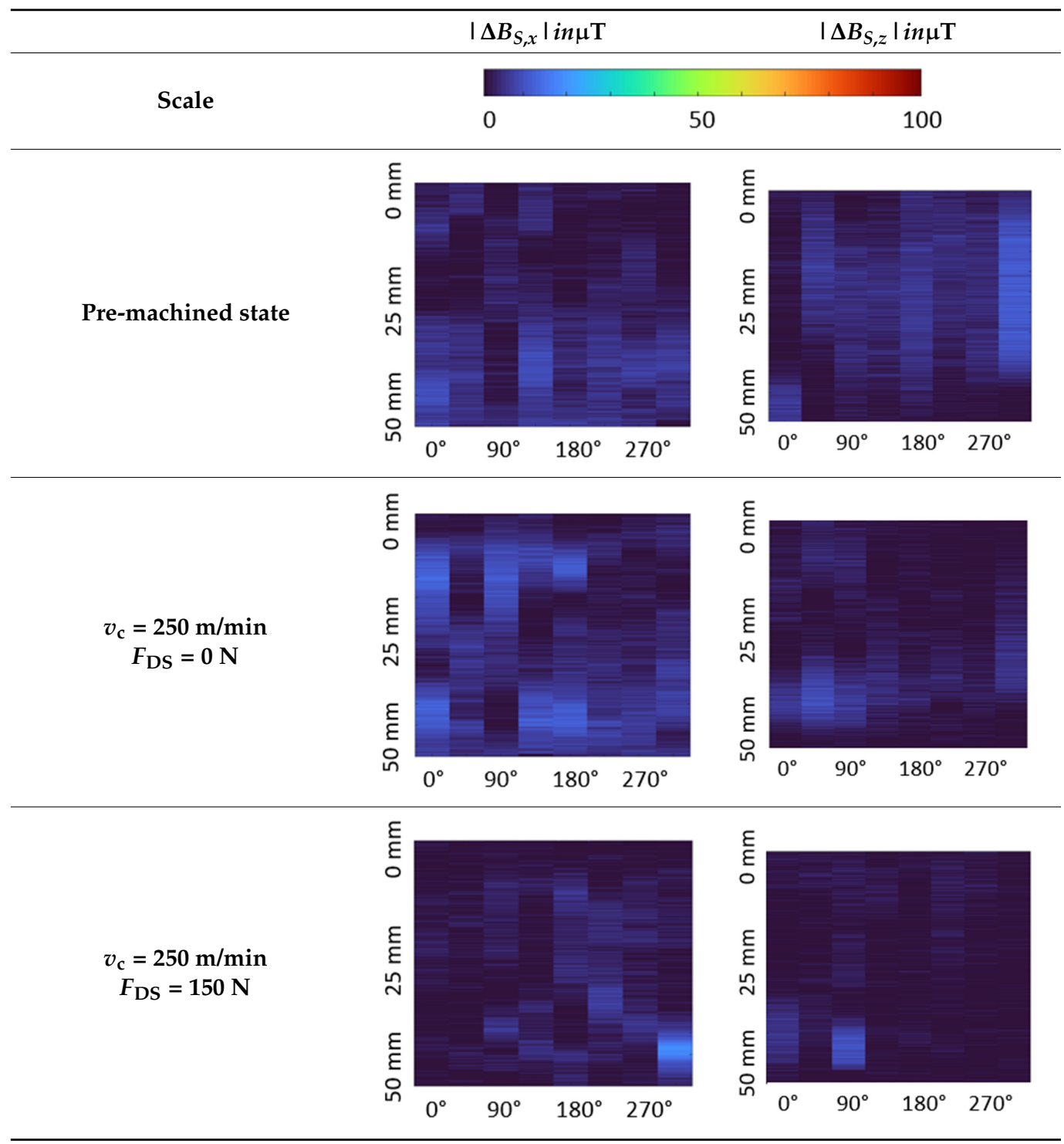

\subsection{Linkage between Results}

Linear correlation coefficient $r$ is used to discover the strength of dependency of one characteristic on another. The possible range of $r$ is between -1 and +1 . The closer $|r|$ is to 1 , the stronger is the correlation. This coefficient is determined in the following equation, where $x_{i}$ and $y_{j}$ represent data points and $\bar{x}$ and $\bar{y}$ represent mean values of correlating amounts of $x$ and $y$ [17]:

$$
r=\frac{\sum\left(x_{i}-\bar{x}\right) \cdot\left(y_{i}-\bar{y}\right)}{\sqrt{\sum\left(x_{i}-\bar{x}\right)^{2} \cdot\left(y_{i}-\bar{y}\right)^{2}}}
$$

Correlation coefficients in this study were on a high level, so $r \geq 0.75$ or even $r \geq 0.95$ occurred quite often. On the one hand, there are probably several strong correlations, but on the other hand, each dataset only consists of three or four data points, which makes it easier to acquire high $r$-values than it would be in the case of large datasets.

However, if the absolute value of the correlation coefficient of two datasets is close to 1 , it can be assumed that there is a mathematical relation at the bottom of the data. Calculations were separated into two groups according to machining technologies. Specimens which were turned exclusively showed that magnetic and other properties are in fact linked. 
Cutting speed correlated with magnetic leakage fluxes (maxima, mean values, and RMS values of $x$ - and $z$ - vector components), machining forces and temperature, remanent MBN signals, and incremental permeabilities. Machining temperature, represented by thermoelectric voltages and currents, seems to control magnetic properties in general. Normal and shear residual stresses did not correlate with many other characteristics. In-process forces and temperatures seem to mostly affect the residual stress state. In contrast, the influence on the magnetic properties was minor. One exception is the correlation between coercive field strength and tangential shear residual stress, with $r_{H C M, \sigma t s}=0.77$ and $r_{H C \mu, \sigma t s}=0.78$. Additionally, maximum MBN signals seem to depend on normal and shear components of tangential residual stresses, which fits the direction of cutting.

The combination of turning and diamond smoothing also showed a strong dependency of several characteristics, such as surface roughness, grain size, and magnetic properties, on DS force. As mentioned above, processes of metal forming provoke microstructural changes. The relationship between magnetic properties, such as magnetic hardness, leakage fluxes, and plasticity, controlling grain sizes, was obvious. Leakage fluxes also correlated with magnetic properties gained by 3MA. DS showed stronger correlations between residual stresses and the other properties. Every kind of residual stress examined here, except for shear stresses in the direction of feed motion, had a strong link to all kinds of magnetic properties, in-process temperatures, forces, and grain size. Forming obviously changed many properties of a materials surface layer at once, in a consistent way.

Correlations showed a coherent and consistent linkage between plausible theories such as magnetic deviations due to plastification, and helped to qualify that the experimental methods used complement one another in order to approach a bigger picture of the whole system.

\section{Conclusions and Future Work}

Based on the results discussed in this paper, the following points conclude the main findings:

- Whether machining or transport have a bigger impact on magnetic properties depends on the regarded characteristics. SHPM analyses have shown that machining, storage, and transport influence some characteristics of magnetic leakage flux differently. In some cases, machining predominates transport and storage. In other cases, it is the other way around.

- MFM contributes to a microstructural assessment but does not display magnetic domains on the inspectable scale.

- Diamond smoothing seems to provoke magnetic hardening of the specimens, whereas turning has an effect tending towards magnetic softening. Loading conditions possibly cause variations in magnetic properties, even if their magnitude can be regarded secondary. An alternative explanation would be that DS causes magnetic hardening due to cold working mechanisms.

- Turning causes bigger changes in the magnetic leakage flux state than DS does, but DS cannot be considered in isolation, because turning is always the pre-process. Machining demagnetized specimens, and repetition of the same experiments might clarify this issue.

- Transport tends to demagnetize the specimens, and only maxima can be affected in different ways. Consequently, magnetic homogeneity is reduced, or at least changed.

- Storage has a much smaller impact than transport. Its effect leads to a demagnetization, where positive and negative leakage flux values slowly head towards zero, but the effect is so weak that there is no relevance in that.

- Correlations showed the impact of machining parameters on magnetic properties. Non-magnetic properties such as grain size also seem to be linked to magnetic ones. Machining forces and temperatures influence the magnetic state of a specimen. Residual stress state depends less on other properties, especially in the case of turning. In DS, residual stress correlates with nearly every other characteristic, except for the 
shear stresses in the direction of the feed motion. Magnetic properties are linked to each other, even if they are acquired by different methods, so correlations contribute to a coherent and consistent view on the whole system.

- All in all, the existence of a degree of deformation caused by machining techniques seems possible. Plastic deformations of different magnitudes within a surface layer could explain the trends in magnetic and general property changes. There is a need for experimental verification in further experiments.

Even if results and conclusions seem plausible, further experiments are required to assure a sufficient confidence level.

In future studies, the objective could be to explore machining influences by varying more than just one parameter in each stage of production. A design of experiments approach might help to cover a large variety of factors and provide a certain confidence level. The occurrence and magnitude of plastic deformation during turning and diamond smoothing could be a crucial but hard to access characteristic, that might explain different impacts of machining types and conditions.

Author Contributions: Conceptualization, H.L. and C.R.; Investigation, O.M.; Methodology, O.M.; Supervision, D.B. and A.S.; Writing-original draft, O.M. and H.L.; Writing-review \& editing, C.R. All authors have read and agreed to the published version of the manuscript.

Funding: This research received no external funding.

Institutional Review Board Statement: Not applicable.

Informed Consent Statement: Not applicable.

Data Availability Statement: Data can be provided by the corresponding author.

Acknowledgments: The authors thank the European Regional Development Fund (ERDF) for supporting their research within the project "SE-ProEng-Systems Engineering zur flexiblen Produktgestaltung". We acknowledge support by the Deutsche Forschungsgemeinschaft (DFG, German Research Foundation) and Saarland University within the funding programme Open-Access Publishing.

Conflicts of Interest: The authors declare no conflict of interest.

\section{References}

1. Clapham, L.; Jagadish, C.; Atherton, D.; Boyd, J. The influence of controlled rolling on the pulse height distribution of magnetic Barkhausen noise in steel. Mater. Sci. Eng. A. 1991, 145, 233-241. [CrossRef]

2. Trapp, K.; Herter, F.; Bähre, D. Magnetic Property Changes by Machining Ferromagnetic Materials. Procedia CIRP 2016, 46, 250-253. [CrossRef]

3. Trapp, K.; Käfer, L.; Bähre, D. Effects of Machining Ferromagnetic Materials on Magnetic Property Changes. In Proceedings of the 43rd North American Manufacturing Research (NAMRC 43), Charlotte, NC, USA, 8-12 June 2015; pp. 721-727. [CrossRef]

4. Tekkaya, A.; Allwood, J.; Bariani, P.; Bruschi, S.; Cao, J.; Gramlich, S.; Groche, P.; Hirt, G.; Ishikawa, T.; Löbbe, C.; et al. Metal forming beyond shaping: Predicting and setting product properties. Ann. CIRP 2015, 64, 629-653. [CrossRef]

5. Brinksmeier, E.; Meyer, D.; Heinzel, C.; Lübben, T.; Sölter, J.; Langenhorst, L.; Frerichs, F.; Kämmler, J.; Kohls, E.; Kuschel, S. Process Signatures-The Missing Link to Predict Surface Integrity in Machining. Procedia CIRP 2018, 71, 3-10. [CrossRef]

6. Weiss, H.A.; Leuning, N.; Hameyer, K.; Hoffmann, H.; Volk, W. Manufacturing efficient electrical motors with a predictive maintenance approach. CIRP Ann. 2019, 68, 253-256. [CrossRef]

7. Leuning, N.; Steentjes, S.; Hameyer, K. Effect of grain size and magnetic texture on iron-loss components in NO electrical steel at different frequencies. J. Magn. Magn. Mater. 2019, 469, 373-382. [CrossRef]

8. Boehm, A.; Hahn, I. Influence of turning on the magnetic properties of electrical steel in the production of electrical machines. In Proceedings of the 2011 IEEE International Electric Machines \& Drives Conference (IEMDC), Niagara Falls, ON, Canada, 14-17 May 2011; pp. 83-88.

9. Senda, K.; Ishida, M.; Nakasu, Y.; Yagi, M. Influence of shearing process on domain structure and magnetic properties of non-oriented electrical steel. J. Magn. Mater. 2006, 304, 513-515. [CrossRef]

10. Makar, J.M.; Tanner, B.K. The effect of plastic deformation and residual stress on the permeability and magnetostriction of steels. J. Magn. Magn. Mater. 2000, 222, 291-304. [CrossRef]

11. Bao, S.; Liu, X.; Zhang, D. Variation of Residual Magnetic Field of Defective U75V Steel Subjected to Tensile Stress: Residual Magnetic Field of Defective U75V Steel under Tensile Stress. Strain 2015, 51, 370-378. [CrossRef] 
12. Honda, T.; Santos, E.C.; Kida, K. Scanning Hall probe microscopy of residual magnetic fields around plastic deformation of Vickers indentations in carbon tool steel (JIS, SKS93). Mech. Mater. 2014, 69, 262-269. [CrossRef]

13. Čilliková, M.; Dubec, J.; Neslušan, M.; Mičietová, A.; Blažek, D. Magnetic Evaluation of Residual Stresses and Structure Transformations Induced in Soft Steel after Turning. Acta Phys. Pol. A. 2014, 126, 60-61. [CrossRef]

14. Klocke, F. Manufacturing Processes 1: Cutting; Springer: Berlin/Heidelberg, Germany, 2011.

15. Gorkunov, E.S.; Zadvorkin, S.M.; Kokovikhin, E.A.; Tueva, E.A.; Subachev, Y.V.; Goruleva, L.S.; Podkopytova, A.V. The effects of deformations by rolling and uniaxial tension on the structure and the magnetic and mechanical properties of armco iron, steel 12X18H10T, and a steel 12X18H10T-Armco Iron-Steel 12X18H10T composite material. Russ. J. Nondestruct. Test. 2011, 47, 369-380. [CrossRef]

16. Baldo, S.; Mészáros, I. Effect of cold rolling on microstructure and magnetic properties in a metastable lean duplex stainless steel. J. Mater. Sci. 2010, 45, 5339-5346. [CrossRef]

17. Roe, B.P. Probability and Statistics in the Physical Sciences; Springer International Publishing: Cham, Switzerland, 2020. 